\title{
Bandwidth Analysis of Smart Meter Network Infrastructure
}

\author{
Kartheepan Balachandran*, Rasmus L. Olsen*, Jens M. Pedersen* \\ *Networking and Security section, Department of Electronic Systems \\ Aalborg University, Fredrik Bajers Vej 7, DK-9220 Aalborg Oest, Denmark \\ kba@es.aau.dk, rlo@es.aau.dk, jens@es.aau.dk
}

\begin{abstract}
Advanced Metering Infrastructure (AMI) is a network infrastructure in Smart Grid, which links electricity customers to utility company. Compared to a network where different amount of data is send, AMI network sends small amount of data at different time intervals. This network enables smart services by making it possible for the utility company to get an overview of their customers power consumption and also control devices in their costumers household e.g. heat pumps. The problem investigated in this paper is what bandwidth requirements can be expected when implementing such network to utilize smart meters and which existing broadband network technologies can facilitate this smart meter service. Initially, scenarios for smart meter infrastructure are identified. The paper defines abstraction models which cover the AMI scenarios. A general overview of the bandwidth requirements are analysed. For this analysis assumptions and limitations are defined. The results show the bandwidth provided by GPRS technology is sufficient. However, from a delay point of view, GPRS has its limits, therefore, xDSL or $3 G$ can be used for faster transfer but will rarely utilize the available bandwidth from these technologies. The aggregator which is responsible to collect all the data from the different smart meters can become a bottleneck as the aggregated amount of data is high, thus the bandwidth for the data concentrator has to be high. These results are interesting to look at, as they show there are different bandwidth requirements from a smart meter and aggregator point of view and that, the aggregator is a vital element in the network and it has to be studied further.
\end{abstract}

Keywords-smart grid, AMI, advanced metering infrastructure, bandwidth requirement, AMI scenario

\section{INTRODUCTION}

The next generation electric infrastructure is called the Smart Grid. It is an advanced electrical infrastructure with bidirectional flow of electricity and information. The smart grid gives the utility companies and the consumers more flexibility to produce and consume electricity [1].

The smart meter is a device deployed at the customer and can vary in model and features. The primary goal of a smart meter is to monitor and aggregate the power consumption information of all devices in the house and send the consumption data to the utility company. Additionally, some smart meters also provide services, as a home gateway, by giving the customer an overview of the power consumption of each appliance and to schedule on/off time of each device and thereby save power. Some smart meters give access to remotely turn off/on devices e.g. a light which has been forgotten to be turned off when leaving the house.

Smart meter can also give the utility company access to devices at their customer, which can help to shift the load on the grid, this is called direct load control (DLC). The idea behind DLC is to enable access to devices which can have a significant power usage and therefore be controlled to lower power consumption on a the local power grid or when having an overproduction of power, to be able to turn on the devices to take away load on the grid [2]. Devices which can be used to shift load on the grid are called distributed energy resources, (D.E.R), because they are distributed resources and some of them belong to customers [1]. DER's can be electric cars, freezers, heat pumps etc.

To make use of the mentioned features of smart grid and smart meters, it requires some communication network infrastructure to facilitate the information flow. This network is called the advanced metering infrastructure (AMI) and is the focus of this paper. The network is usually taken for granted. However, it is important to analyse the requirements for the network in order to be able to choose what kind of communication technology can facility the needs and thereby avoiding to design a network infrastructure which is too expensive or is performing very poorly.

\section{CONTRIBUTION}

To utilise the advantages of the smart grid requires the communication network to work reliably and secure. Several project-proposals for AMI have been made using different communication technologies [3] [4] [5]. However, there is a lack of network requirements estimation in order to get an overview of what to expect from a network planned to be deployed for AMI.

The amount of data which will be sent from a smart meter has to be analysed in order to determine an appropriate and feasible network technology to be used whether wireless or wired. Smart grid or AMI does not have standardised architecture, thus many different implementations exist and the requirements for the communication infrastructure depends on the implementation. Therefore, AMI scenarios chosen in this paper cover the most common ideas of AMI and the bandwidth requirements are analysed for these scenarios.

The paper will cover following:

- Give a general overview of the bandwidth requirements based on analysis of smart grid scenarios presented in this paper.

- The results presented shows how much bandwidth is needed and which communication technologies can be used to cover these requirements.

- A discussion of the achieved results, limitation of the technologies and the role of aggregation point in AMI networks will be addressed. 
The paper is constructed in following way: First the scenarios and assumptions are defined on which the analysis will be based on. Then the results are presented and finally a discussion of the results and assumptions and a conclusion followed with future work.

\section{Methodology}

The first step will be to define abstraction models which cover AMI scenarios. The models will be based on how AMI scenarios are discussed in the smart grid community. The bandwidth requirement analysis will be based on these scenarios.

The second step states the assumptions made for the analysis of the scenarios. This part will present the assumed network condition, the data transfer protocol, which network protocols are considered and the assumption of the technologies which can be used in smart grid. The analysis will not take into account every detail of the network, therefore, the analysis will only give a rough overview of the bandwidth requirements when analysing the AMI bandwidth requirement, however, section VII will discuss the assumptions and the results which are affected by this.

To calculate how much bandwidth is required four parameters are necessary to be known. How often the smart meter samples the power usage and what is the size of this sample. This is defined as sample interval $S$ and sample size $\alpha$. Secondly how often the smart meter transmit the monitored data and the size of the data, are defined as transfer interval $T$ and transfer size $\beta$, respectively.

The third step is to calculate how much bandwidth is required for one household to transfer data by a smart meter while the sampling interval and the transfer interval are varied. Based upon these estimations the technologies can be evaluated suitable for the defined scenarios. The aggregators bandwidth requirement is also addressed because it has to aggregate data from many households. This is done by scaling the bandwidth consumption with no. of household in a city taken from Statistics Denmark [6].

\section{SCENARIOS}

The scenarios which will be used in smart grid can generally be described as following. Scenario 1, is shown in Figure 1. It shows the smart meter is connected directly to the DSO. In Scenario 1 the household has a smart meter monitoring the total power consumption and transfers the data to the DSO's aggregator.

\begin{tabular}{|c|c|c|}
\hline $\begin{array}{c}\text { TSO } \\
\text { Scada system }\end{array}$ & T, $\beta$ DSO & Smart Meter \\
\hline
\end{tabular}

Fig. 1: The figure shows AMI scenario 1, where metering data are sent with transfer interval $T$ and transfer data size $\beta$

The second scenario, shown in Figure 2, can be either an agent or the DSO who provides smart meter services. The figure shows an AMI scenario where an agent can act as middleman connecting the DSO and the customers. This way the agent has the responsibility to maintain and offer various services to the utility customers and the DSO. In this model there is two way communication which enables control of energy resources for load balancing purpose. Furthermore, it gives the utility company a detailed overview of the power consumption of connected appliances in the home by having sensors to measure the power plugs.

In scenario 2 the household will have a smart meter connected to a number of sensors measuring detailed power consumption of connected appliances. The amount of sensors can be any number and depends on the size of the house and number of devices to be monitored. In this scenario it is chosen to be 10 sensors. Control application is not considered in this paper.

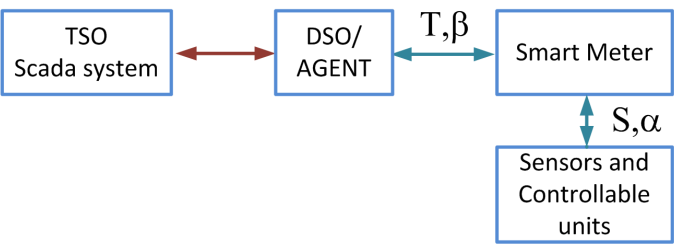

Fig. 2: The figure shows AMI scenario 2, where sensors send samples with sample interval $S$ and sample size $\alpha$ to the smart meter and the smart meter sends accumulated consumption data according to scenario 1

A part of the scenario definition is also to define how often the smart meter and the sensors monitor or samples the power consumption and how often the monitoring data are transferred. The variables are described in section III and are shown in table I. The purpose is to explore how much bandwidth will be required in different scenarios if a smart meter varies the sample and transfer interval.

\section{TABLE I}

\begin{tabular}{|c|c|c|c|c|c|}
\hline \multicolumn{7}{|c|}{ Sampling and Transfer rate } \\
\hline Sample interval $S_{X}$ & $0.5 \mathrm{sec}$ & $2 \mathrm{sec}$ & $30 \mathrm{sec}$ & $1 \mathrm{~min}$ & $5 \mathrm{~min}$ \\
\hline Transfer interval $T_{Y}$ & $1 \mathrm{sec}$ & $4 \mathrm{sec}$ & $60 \mathrm{sec}$ & $5 \mathrm{~min}$ & $15 \mathrm{~min}$ \\
\hline
\end{tabular}

To sum up the scenarios are as follows:

- Scenario 1: Data transfer with data from only the smart meter

- Scenario 2: Data transfer with data from the smart meter and sensors measuring power usage of connected appliances

In both scenarios the sampling of data and transferring the accumulated data from the smart meter to the aggregator are according to Table I. The table defines different sampling and transferring schemes to the above two scenarios.

The aggregator receives data from both scenarios. It must be able to handle multiple connection and store and forward information from multiple smart meters. The aggregator can become a bottleneck in AMI networks and has to be investigated to see how much data it has to handle. In Table II the number of households is listed for the biggest cities in Denmark. It is assumed every household will have a smart meter. The case study will be to assign an aggregator for each city.

\section{ASSUMPTIONS}

In this section the assumptions will be described which the analysis will be based upon, but initially a brief discussion is made of the wireless and wired technology that are considered. 


\section{TABLE II}

\begin{tabular}{|l|c|}
\hline \multicolumn{2}{|l|}{ Households in Denmark 3 biggest cities in 2013 [6] } \\
\hline City & No. of households \\
\hline Copenhagen & 285.281 \\
\hline Aarhus & 151.738 \\
\hline Aalborg & 100.152 \\
\hline
\end{tabular}

\section{A. Wireless Technologies}

For wireless technologies GPRS and $3 \mathrm{G}$ is considered. GPRS has a high coverage area but a lower bandwidth. 3G is widely spread and gives higher bandwidth. The available bandwidth on both technologies is limited by the distance to the antenna and the number of users connected.

\section{B. Wired Technologies}

For wired solution xDSL or Coax connection and PLC connection are considered. xDSL or cable connection is already existing in most household, however, the drawback is; it is a shared connection meaning the bandwidth will be shared with the user for different purposes. Power Line Communication (PLC) has an advantage to use the existing power line infrastructure, but the drawback with PLC is; it is a very noise medium where the real bandwidth is limited.

\section{Scenario Assumptions}

The data from a smart meter is transferred to an aggregator, this aggregator can be placed at the DSO, agent or stand alone. Nevertheless, only the network connection from the smart meter to the aggregator is analysed. This connection can vary with different technologies as it depends on the connection the household gets installed to communicate with the aggregator.

- The access network is the network connection from the smart meter to the aggregator. The connection from the smart meter is assumed to have the lowest available connection compared to the backbone. Therefore, the bandwidth from the access technology is considered as the maximum bandwidth available to be used to send data to the aggregator.

- The network connection is considered to be perfect which means that there is no delay, except the delay imposed by the access technology. Packets are not lost, thus retransmissions are not considered.

- All smart meters are synchronised and will push data at the same time. This assumption represents a worst case scenario and will be discussed later.

- The aggregator has an infinite buffer capable to receive all data transferred from the smart meters and process it before the next amount of data arrives.

- Wireless access networks are considered not to be limited in capacity even though it is a shared resource. This means each connection will have the same amount of bandwidth and served simultaneously.

- The overhead protocols included will be UDP over IP. The phy-layer protocol depends on the medium transferred. The overheads imposed by the technology are not considered. However, the smart meter will be using Ethernet when connecting to DSL modem. Ethernet overhead is therefore included in the bandwidth calculation as a physical layer overhead.

\section{Data Sizes}

The size of the data transferred depends on the system implemented. Table III present the datafields and the data sizes.

\section{TABLE III}

\begin{tabular}{|l|c|c|c|}
\hline \multicolumn{4}{|c|}{ Data field and size (bits) } \\
\hline Unit & Id & Timestamp & Data \\
\hline smart meter \& each sensors & 64 & 32 & 128 \\
\hline
\end{tabular}

The parameters and the data presented in Table III are only minimum of information required from the smart meter and the sensors. The Data size of 128 bit represent 8 bytes of numerical measurement data and 8 bytes of meta data. The last is due to cover that different smart meter might also record different information.

The protocol used to pack and transfer the data are not specified as this also depends on different standards and products. To cover different system and information which are not considered, the amount of data generated to be transferred is multiplied with a overhead factor 10 and called the main overhead, $O H_{\text {main }}$.

Furthermore, when multiple sensor data is added to the smart meter each sensor data is multiplied with a overhead of factor $3, \mathrm{OH}_{\text {bundle }}$ to cover additional information. When working with embedded systems it is good to bundle data as this lowers the amount of data transferred and the load on the aggregator side when reading the incoming data.

Based on these assumption the equation to calculate the bandwidth requirement are described as follows:

$$
\begin{array}{cc}
\overline{\alpha_{S_{X}}} & =\gamma_{\text {mtrdata }} \cdot\left(O H_{\text {main }}+O H_{\text {bundle }}\left(\left(\frac{T_{Y}}{S_{X}}-1\right)+n_{\text {snsr }}\right)\right) \\
\overline{\beta_{T_{Y}}} & =\quad \alpha_{S_{X}}+\frac{\alpha_{S_{X}}}{M T U} \cdot(U D P+I P+E T H E R N E T)_{h d r s z e}
\end{array}
$$

where

$$
\begin{aligned}
\overline{\alpha_{S_{X}}} & =\text { avg. data size for sample interval } X \\
\overline{\beta_{T_{Y}}} & =\text { avg. data size for transfer interval } Y \\
T_{Y} & =\text { transfer interval } Y \\
S_{X} & =\text { sample interval } X \\
n_{\text {snsr }} & =\text { no of sensors } \\
\gamma_{\text {mtrdata }} & =\text { size of meter data from table III } \\
O H_{\text {main }} & =\text { overhead factor } 10 \text { for main packet to be transferred } \\
O H_{\text {bundle }} & =\text { overhead factor } 3 \text { for bundling continues data } \\
M T U & =\text { Maximum Transfer Unit (1500 bytes) } \\
h d r s z e & =\text { Minimum header size of UDP, IP and Ethernet }
\end{aligned}
$$

\section{REsults}

This section presents the results based on the scenarios and assumptions introduced previously. Two tables are presented for each scenario. First table presents the accumulated amount of data before it is sent to the aggregator. E.g. How much data is collected during 5 minutes interval including overhead data for transferring to aggregator.

The second table presents the average bandwidth requirement when transferring the data at different transfer intervals. E.g. How much avg. bandwidth is required to transfer over a period of $5 \mathrm{~min}$. before the next set of data is ready to be transferred. Finally, a worst case scenario is presented for the aggregator. 
The first results presented in Table IV shows the bandwidth required when only one smart meter is transferring the total power consumption data for a household. The overhead for transferring the generated data is a part of the data and shown as a percentage it constitute to the data. Table $\mathrm{V}$ shows the average bandwidth required to transfer the accumulated meter data.

\section{TABLE IV}

\begin{tabular}{|l|c|c|c|c|c|}
\hline \multicolumn{5}{|c|}{ Data to be transferred and percentage of overhead } \\
\multicolumn{5}{|c|}{ for 1 smart meter $\left.\begin{array}{c}\text { byte } \\
\%\end{array}\right]$} \\
\hline \multicolumn{5}{|c|}{ Transfer interval } \\
\hline Sample interval & 1 sec & 4 sec & 60 sec & 5 min & 15 min \\
\hline \multirow{2}{*}{$0.5 \mathrm{sec}$} & 422 & 926 & 10682 & 52568 & 157254 \\
& 13,74 & 6,26 & 3,80 & 3,75 & 3,73 \\
\hline \multirow{2}{*}{$2 \mathrm{sec}$} & 0 & 422 & 2832 & 13318 & 39504 \\
& 0 & 13,74 & 4,10 & 3,92 & 3,82 \\
\hline \multirow{2}{*}{$30 \mathrm{sec}$} & 0 & 0 & 422 & 1094 & 2832 \\
& 0 & 0 & 13,74 & 5,30 & 4,10 \\
\hline \multirow{2}{*}{$1 \mathrm{~min}$} & 0 & 0 & 338 & 674 & 1514 \\
& 0 & 0 & 17,16 & 8,61 & 3,83 \\
\hline \multirow{2}{*}{$5 \mathrm{~min}$} & 0 & 0 & 0 & 338 & 506 \\
& 0 & 0 & 0 & 17,16 & 11,46 \\
\hline
\end{tabular}

\section{TABLE V}

\begin{tabular}{|l|c|c|c|c|c|}
\hline \multicolumn{6}{|c|}{ Average bandwidth required at different transfer intervals } \\
for 1 smart meter $[\mathrm{bps}]$ \\
\hline & \multicolumn{5}{|c|}{ Transfer intervals } \\
\hline Sample interval & $1 \mathrm{sec}$ & $4 \mathrm{sec}$ & $60 \mathrm{sec}$ & $5 \mathrm{~min}$ & $15 \mathrm{~min}$ \\
\hline $0.5 \mathrm{sec}$ & 3376 & 1852 & 1424,3 & 1401,8 & 1397,8 \\
\hline $2 \mathrm{sec}$ & 0 & 844 & 377,6 & 355,15 & 351,15 \\
\hline $30 \mathrm{sec}$ & 0 & 0 & 56,27 & 29,17 & 25,17 \\
\hline $1 \mathrm{~min}$ & 0 & 0 & 45,07 & 17,97 & 13,46 \\
\hline $5 \mathrm{~min}$ & 0 & 0 & 0 & 9,01 & 4,50 \\
\hline
\end{tabular}

The results (Table IV) for scenario one shows, when sampling every $0.5 \mathrm{sec} 422$ bytes have to be transferred every $1 \mathrm{sec}$. From the generated data $13,74 \%$ constitute to overhead which means the generated data do not utilise MTU of 1500 bytes for Ethernet. In other words, the amount of data accumulated compared to the overhead is not effective.

Looking at another case where it is sampled every $1 \mathrm{~min}$ and transferred every $5 \mathrm{~min}$, it can be seen that 674 bytes of data has to be transferred which is still less than MTU of an Ethernet frame. In other words, every 5 minute accumulated data will be transferred by one Ethernet packet.

The highest amount of data generated is the scenario where data is sampled every $0.5 \mathrm{sec}$ and transferred every $15 \mathrm{~min}$. The generated amount of data is 157254 bytes (14,8 KBytes) where $3.73 \%$ constitute to overhead. In this scenario more data are transferred compared to overhead. It is noticeable when different system factors and high sampling time is taken into account, the amount of data accumulated by one smart meter over 15 minutes is still low compared to how much it will utilize the bandwidth available by broadband technology.

Table $\mathrm{V}$ shows the required bandwidth is low to transfer the different amount of accumulated data from Table IV. The highest demand is seen when data has to be transferred every second, then a bandwidth of 3376 bits/s is required. However, over 15 minutes transfer interval, 157254 bytes of data require an avg. bandwidth of $1398 \mathrm{bit} / \mathrm{s}$ or $1,3 \mathrm{Kbit} / \mathrm{s}$. With lower sampling intervals like $1 \mathrm{~min}$ or $5 \mathrm{~min}$ the lowest bandwidth required becomes 4,50 bits/s.

The results presented in Table VI, show the amount of data generated to be transferred in scenario 2, where data from a smart meter and 10 sensors which are monitoring appliances, are aggregated together. Again, the sampling time and transfer times are varied according to Table I. Table VII shows the average bandwidth requirements at different transfer intervals for scenario 2 .

\section{TABLE VI}

\begin{tabular}{|c|c|c|c|c|c|}
\hline \multicolumn{4}{|c|}{ for 1 smart meter and 10 sensors } & $\begin{array}{l}\text { of ove } \\
{\left[\begin{array}{c}b y t e \\
0\end{array}\right.}\end{array}$ & \\
\hline & \multicolumn{5}{|c|}{ Transfer time } \\
\hline Sample time & $1 \mathrm{sec}$ & $4 \mathrm{sec}$ & $60 \mathrm{sec}$ & $5 \mathrm{~min}$ & $15 \mathrm{~min}$ \\
\hline \multirow{2}{*}{$0.5 \mathrm{sec}$} & 1262 & 1824 & 11580 & 53466 & 158152 \\
\hline & 4,60 & 6,36 & 4,01 & 4,08 & 3,74 \\
\hline \multirow{2}{*}{$2 \mathrm{sec}$} & 0 & 1262 & 3730 & 14216 & 40344 \\
\hline & 0 & 4,60 & 4,66 & 5,83 & 3,74 \\
\hline \multirow{2}{*}{$30 \mathrm{sec}$} & 0 & 0 & 1262 & 1992 & 3730 \\
\hline & 0 & 0 & 4,60 & 3,83 & 4,66 \\
\hline \multirow{2}{*}{$1 \mathrm{~min}$} & 0 & 0 & 1178 & 1514 & 2412 \\
\hline & 0 & 0 & 4,92 & 6,52 & 4,81 \\
\hline \multirow{2}{*}{$5 \mathrm{~min}$} & 0 & 0 & 0 & 1178 & 1346 \\
\hline & 0 & 0 & 0 & 4,92 & 4,31 \\
\hline
\end{tabular}

TABLE VII

\begin{tabular}{|l|c|c|c|c|c|}
\hline \multicolumn{6}{|c|}{$\begin{array}{c}\text { Average bandwidth required at different transfer intervals } \\
\text { for 1 smart meter }[\mathrm{bps}]\end{array}$} \\
\hline & \multicolumn{6}{|c|}{ Transfer time } \\
\hline Sample time & $1 \mathrm{sec}$ & $4 \mathrm{sec}$ & $60 \mathrm{sec}$ & $5 \mathrm{~min}$ & $15 \mathrm{~min}$ \\
\hline $0.5 \mathrm{sec}$ & 10096 & 3648 & 1544 & 1425,8 & 1405,8 \\
\hline $2 \mathrm{sec}$ & 0 & 2524 & 497,33 & 379,09 & 358,61 \\
\hline $30 \mathrm{sec}$ & 0 & 0 & 168,27 & 53,12 & 33,16 \\
\hline $1 \mathrm{~min}$ & 0 & 0 & 157,07 & 40,37 & 21,44 \\
\hline $5 \mathrm{~min}$ & 0 & 0 & 0 & 31,41 & 11,96 \\
\hline
\end{tabular}

The scenario where it is sampled every $0.5 \mathrm{sec}$ and transferred every $1 \mathrm{sec}, 1262$ bytes have to be transferred (Table VI). The amount of overhead in this case is $4,60 \%$ which is 3 times less than previous scenario. This difference is because of the addition of sensor data in this scenario. Basically more data is transferred which is utilizing MTU of a ethernet frame.

When the system samples every $1 \mathrm{~min}$. and transfer every 5 min. the system generates 1514 bytes of data and has the highest amount of overhead $6.52 \%$ compared to the other results in this scenario. Generally, the overhead data is less in this scenario. The highest amount of data generated is 158152 bytes or 154 Kbytes when sampling every $0.5 \mathrm{sec}$ over a period of 15 minutes. With a GPRS connection of 171,23 kbps it will take around $8 \mathrm{sec}$ and with a transfer interval of 15 minutes this amount of data can easily be transferred with GPRS including the delays which is not accounted for.[7]

Table VII shows the bandwidth required to transfer the accumulated data shown in Table VI for scenario 2. The average 
bandwidth requirement are higher in scenario 2 compared to scenario 1 due to the extra data from the sensors, however, it is not a big difference. The highest demand is $10096 \mathrm{bits} / \mathrm{s}$ for transferring data every second. Generally, shorter transfer interval requires higher bandwidth. Over 15 minutes interval the generated data to be transferred is highest, however, the bandwidth required is lower due to the long interval for the data to be transferred.

The table (Table VII) shows, strictly from a bandwidth point of view, GPRS connection can facilitate smart meter system with a more detailed overview of the power consumption. However, when low latency is a requirement where data has to be transferred less than a minute, GPRS will become a problem. Therefore, broadband technologies as $\mathrm{xDSL}$ or $3 \mathrm{G}$ can be used instead, but the results are pointing that the existing technologies will not have problems with these bandwidth demands. The challenge will be to handle short term flow burst generated by smart meters.

The results presented so far are showing how much data is generated and how much bandwidth is required to transfer smart meter measurement with and without additional sensor data. The next results presented in Table VIII is how much data will the aggregator receive in worst case scenario. A worst case scenario will be, if all smart meters are sending power measurement data at the same time. Based on the scenarios defined, the worst case scenario will be smart meters with 10 sensors sending data every 15 minutes at different sample intervals because this scenario generates highest amount of data.

Another example of a worst case scenario is when sending data every second because this requires the aggregator to open and close many connections or by letting the smart meters stream the data by having an open connection. However, it is not a realistic scenario. Therefore, it is focused on how much data the aggregator has to handle if it receives bursts of data every 15 minutes. The number of smart meters correspond to the amount of household shown in Table II and the amount data generated is from scenario 2 .

\section{TABLE VIII}

\begin{tabular}{|l|c|c|c|c|c|}
\hline \multicolumn{6}{|c|}{$\begin{array}{c}\text { Amount of data (in MByte) an aggregator receives every } 15 \text { min. } \\
\text { at different sampling rates }\end{array}$} \\
\hline & \multicolumn{6}{|c|}{ Sampling times } \\
\hline no. of households (City) & $0.5 \mathrm{sec}$ & $2 \mathrm{sec}$ & $30 \mathrm{sec}$ & $1 \mathrm{~min}$ & $5 \mathrm{~min}$ \\
\hline 285281 (Copenhagen) & 43028 & 10976 & 1014,8 & 656,22 & 366,2 \\
\hline 151738 (Aarhus) & 22886 & 5838,1 & 539,76 & 349,04 & 194,78 \\
\hline 100152 (Aalborg) & 15105 & 3853,4 & 356,26 & 230,38 & 128,56 \\
\hline
\end{tabular}

From Table VIII the highest amount of data received by the aggregator in one burst is $43028 \mathrm{MB}$ which is a lot of data at once. The amount of data is generally high for low sampling time but at $1 \mathrm{~min}$. and $5 \mathrm{~min}$. sampling time it becomes more manageable. Nevertheless, this table clearly shows the aggregator can easily become the bottle neck. Many smart meters sending at the same time can easily put down the aggregator. Therefore, some kind of smart scheduling is required. Instead of the smart meter pushing data to the aggregator it will be smarter to let the aggregator request data from the house holds and thereby control the incoming data. From a bandwidth requirement perspective the aggregator needs atleast an avg. download connection of $401 \mathrm{Mbit} / \mathrm{s}$.

\section{DISCUSSION}

This section will discuss the assumptions and the results achieved in this paper. The results are strictly correlated to the assumptions made. The assumptions do not take into account all different aspect of network constraints e.g. delay in the network. Nevertheless, the results presented give an indication of requirements for an AMI network. The case studies have given some insight in what to consider when analysing AMI systems.

Having a perfect network does not match the real world, however, it eases up the estimation calculations to get a rough picture of what is needed. Using existing broadband infrastructure imposes different delays. In this paper it is assumed that there are no other delays than the delay caused by the bandwidth given by the access technology. This includes the following.

First of all, the data is going through the internet, thus, the packet will be exposed to different routing delays on its way to the aggregator. The packet can also be lost which gives retransmissions delays. These factors increase the complexity of the bandwidth requirement estimation and will be included in further study.

Secondly, the delay imposed by the bandwidth available from a technology, is a question of how fast the smart meter can transfer the data onto the access medium. Therefore, it can be called a saturation delay on phy-layer and the question can be asked, if this delay is enough to be considered for transferring data? This is assumed to be enough for this analysis, however, more detailed analysis will be done using simulation.

Wireless systems are assumed to have full capacity for each connection. However, a shared medium like an antenna will impose delays. The performance of a technology depends on how busy it is. It increases the complexity of the scenarios to include how resources are prioritized for each smart meter together with other usage. Thus, is a further work to investigate.

It is assumed that all smart meters are synchronised and push data to the aggregator and it is capable to receive all the data which arrives in bursts. These assumptions have given results that indicate that the aggregator may become overloaded. Thus, it is preferred to let the aggregator schedule and request data from the smart meters. The case study also shows that one aggregator for each major city is not efficient and should be distributed to multiple aggregators. The processing latency of the receiving data is not considered. This can also impose a bottleneck and not only a low bandwidth connection.

The data which are sent from a smart meter are bundled and transferred with UDP. Thus, if some of the segmented packets are not received the whole collected data for an interval will be corrupted and not usable. Even though the amount of data each smart meter generates is low, the accumulated data from all smart meters can overload the aggregator, thus a reliable connection oriented protocol like TCP is preferred.

Furthermore, Ethernet header is used as an example to include some sort of overhead to represent the overhead of the access medium. In a shared broadband connection different overheads are added to the transferred data. Each access medium has its own protocol. One for xDSL another for GSM etc. This is not included in this paper. Adding the right access protocol will only increase or decrease the amount of data size with a small percentage. 
The data sizes used in this paper will in reality vary from system to system. Some smart meter systems generate even less data than anticipated and other systems can generate more data. However, this paper investigates a worst case scenario and therefore, extra overhead factor are included in the case study. With the extra overhead, the amount of data generated from each smart meter is still considered low compared to bandwidth available from the broadband technologies including wireless technologies as GPRS.

\section{CONCLUSION}

The paper has analysed bandwidth requirements for AMI scenarios in smart grid. The analysis is based on some strong assumptions with perfect network conditions and the scenarios are defined by general observations of AMI solutions. Most of the smart grid systems are low data generating systems which the scenarios represent.

A worst case scenario analysis shows the smart meter can generate 158152 byte or 154 KByte of data if sampled every $0.5 \mathrm{sec}$ over a $15 \mathrm{~min}$ interval. From a network coverage point of view a GPRS connection of $171,28 \mathrm{kbps}$ can handle this amount of data, however, it will take few seconds to transmit. In this analysis it will take $8 \mathrm{sec}$ assuming perfect network condition. Other scenarios give a lower bandwidth requirements which indicate a GPRS connection is sufficient for Smart Meter reading in AMI network. However, if the data delivery time requirements becomes less the 60 seconds GPRS will not be feasible, because in real world delays greater than a minute can arise using GPRS.

Because of the low amount of data any faster technologies including GPRS can be used for AMI network in terms of bandwidth requirement. However, from a delay requirement more detailed use cases has to be investigated with different timing requirements. Nevertheless, based on the scenarios investigated in this paper it can be concluded any technology faster than GPRS will be able to handle timing requirements where data has to be transferred every $60 \mathrm{sec}$ or above.

The aggregator which collects data from all smart meters can be expected to become a bottle neck. A worst case study shows if around 285000 smart meters sends data at once, the aggregator will receive up to 43 GByte of data. Other scenarios also show up to 128 MByte of data which is manageable. The case studies made in this paper assume the smart meters push the data to the aggregator. However, it is be more feasible to let the aggregator request data from the smart meter and thereby be able to schedule the arrival of data. From a bandwidth requirement, a worst case study shows $401 \mathrm{Mbps}$ download connection is needed.

To sum up; the achieved results show the amount of data generated by smart meters is low. GPRS and faster communication technologies will be able to handle AMI networks with delay requirements from 1 minute and up. The paper also shows that the aggregator can become a bottle neck due to the amount of smart meters it has to handle.

\section{FUTURE WORK}

The next step will be to simulate the presented scenarios in $\mathrm{OMNeT}++$ to verify the estimated results. Furthermore, an in-depth analysis of existing smart meter system will also be done where security and privacy is investigated. Information dynamics on the aggregator will also be investigated using markov-models and mismatch probability.
ACKNOWLEDGMENT

This project is funded by The European Regional Development Fund.

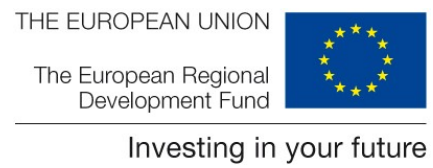

\section{REFERENCES}

[1] W. Wang, Y. Xu, and M. Khanna, "A survey on the communication architectures in smart grid." Computer Networks, vol. 55, no. 15, pp. 3604-3629, 2011. [Online]. Available: http://dblp.unitrier.de/db/journals/cn/cn55.html

[2] J. Byun, I. Hong, B. Kang, and S. Park, "A smart energy distribution and management system for renewable energy distribution and contextaware services based on user patterns and load forecasting," Consumer Electronics, IEEE Transactions on, vol. 57, no. 2, pp. 436 -444, may 2011.

[3] F. Bliek, A. van den Noort, B. Roossien, R. Kamphuis, J. de Wit, J. van der Velde, and M. Eijgelaar, "Powermatching city, a living lab smart grid demonstration," in Innovative Smart Grid Technologies Conference Europe (ISGT Europe), 2010 IEEE PES, oct. 2010, pp. 1 -8.

[4] F. Benzi, N. Anglani, E. Bassi, and L. Frosini, "Electricity smart meters interfacing the households," Industrial Electronics, IEEE Transactions on, vol. 58, no. 10, pp. 4487 -4494, oct. 2011.

[5] S. Depuru, L. Wang, V. Devabhaktuni, and N. Gudi, "Smart meters for power grid -; challenges, issues, advantages and status," in Power Systems Conference and Exposition (PSCE), 2011 IEEE/PES, march 2011, pp. 1 -7 .

[6] S. Denmark, "Households," http://www.dst.dk/en/Statistik/emner/husstandefamilier-boern/husstande.aspx, 2013, [Online; accessed 15-August-2013].

[7] J. Mikkonen and M. Turunen, "An integrated qos architecture for gsm networks," in Universal Personal Communications, 1998. ICUPC '98. IEEE 1998 International Conference on, vol. 1, 1998, pp. 403-407 vol.1. 